Series A

I. MATHEMATICA

432

\title{
ON THE IMPOSSIBILITY OF \\ EXTENDING THE RIESZ UNIQUENESS THEOREM TO FUNCTIONS OF SLOW GROWTH
}

BY

K. F. BARTH and W. J. SCHNEIDER

H EL S I K I 1968

S U O M A L I NEN TIEDEAKA T EM I A

doi:10.5186/aasfm.1969.432 
Communicated 10 May 1968 by K. I. Virtanen and Lauri Mrrberg 


\section{INTRODUCTION}

Throughout this paper we shall let $D$ denote the open unit disk and $C$ denote the unit circumference.

First we recall two classical theorems in the theory of the boundary behavior of functions bounded and analytic in $D$.

Fatou radial limit theorem ([9], p. 45). If $f(z)$ is bounded and analytic in $D$, then $\lim f\left(r e^{i f}\right)$ exists for almost all $\Theta$ in $[0,2 \pi)$.

$F$. and M. Riesz uniqueness theorem ([9], p. 47). If $f(z)$ is bounded and analytic in $D$ and if $\lim f\left(r e^{i(}\right)=0$ for a set of $\Theta$ of positive measure in $[0,2 \pi)$, then $f(z) \equiv 0$.

It is well-known ([8], p. 208-209) that the above theorems also hold if $f(z)$ is meromorphic and of bounded characteristic. It also follows from well-known results that if $f(z)$ is normal in the sense of Lehto and Virtanen then the uniqueness theorem holds also (for the sake of completeness we shall include this as Theorem 3). The elliptic modular function shows that even for normal analytic functions there is no Fatou radial limit theorem.

A natural question to ask is whether the hypothesis " $f(z)$ is bounded" in the Fatou theorem can be weakened for analytic functions to " $M(r, f)$ $\left(=\max _{\Theta}\left|f\left(r e^{i \theta}\right)\right|\right)$ grows slower than 'so and so ")?

Recently, after it had been an open question concerning a number of authors ([1], [4], [5]) for several years, MacLane [6] showed that no matter how slowly an unbounded $M(r, f)$ is required to grow the $\lim _{r \rightarrow 1} f\left(r e^{i \oplus}\right)$ may fail to exist for all $\Theta$ (i.e., there is no analog of the Fatou radial limit theorem for functions of slow growth). The main result of this paper is to show that no matter how slowly an unbounded $M(r, f)$ is required to grow the $\lim _{r \rightarrow 1} f\left(r e^{i \theta}\right)$ may be equal to zero for a set of $\Theta$ of measure $2 \pi$ on $[0,2 \pi)$ (i.e., there is no analog of the Riesz uniqueness theorem for functions of slow growth). The proof is based, to a large extent, on a variation of a technique first used by MacLane in [6]. 


\section{PROOF OF MAIN THEOREMS}

Theorem 1. Let $p(r), 0 \leq r<1$ be positive and monotone increasing to $+\infty$ as $r \rightarrow 1$. Then there exists a function $g(z)$, holomorphic in $D$, with the properties:

i) $\lim _{r \rightarrow 1} \operatorname{Re} g\left(r e^{i \theta}\right)=-\infty$ for almost all $\Theta$ in $[0,2 \pi)$,

ii) $M(r, g)<p(r)$ for $0 \leq r<1$.

Proof. Given any $\varepsilon\left(0<\varepsilon<\min \left\{\frac{1}{4}, p(0)\right\}\right)$ choose $\left\{\varepsilon_{n}\right\}$ with the properties:
i) $\varepsilon_{n} \downarrow 0$,
ii) $\varepsilon_{n}<p(0) / 2^{n}$,
iii) $\sum_{n=1}^{\infty} \varepsilon_{n}<\varepsilon$.

Also pick $\left\{\delta_{n}\right\}$ with the properties:
i) $\delta_{n} \downarrow 0$,
ii) $\sum_{n=1}^{\infty} \delta_{n}<1 / 10$.

Let $R\left(\delta_{n}\right)$ be a Cantor set on $C$ with the property that $2 \pi-\delta_{n}<$ meas $R\left(\delta_{n}\right)<2 \pi$.

Let $\tilde{R}\left(\delta_{n}\right)=\left\{z=r e^{i()}: \frac{1}{2} \leq r \leq 1, e^{i \cap} \varepsilon R\left(\delta_{n}\right)\right\}$.

Also let $\left\{\beta_{n}\right\}$ be a sequence of negative numbers such that $\beta_{n} \downarrow-\infty$.

Using Mergelyan's theorem [7], we can find polynomials $g_{n}(z)$ with the properties:

i) $\left|g_{n}(z)-h_{n}(z)\right|<\varepsilon_{n}$ for $|z| \leq \frac{1}{2}$,

ii) $\left|g_{n}(z)-h_{n}(z)\right|<\varepsilon_{n}$ for $z \in \tilde{R}\left(\delta_{n}\right)$.

(Where $h_{n}(z)$ is the function that equals zero for $\quad z \leq 1 / 2$, equals $\beta_{n}$ for $z \in \tilde{R}\left(\delta_{n}\right) \cap\left\{\frac{7}{8} \leq|z| \leq 1\right\}$ and is linear on each segment $\left\{z=r e^{i \ominus}: \frac{1}{2} \leq\right.$ $\left.r \leq \frac{7}{8}, e^{i \Theta} \in R\left(\delta_{n}\right)\right\}$.)

We now choose a sequence of positive integers $\left\{\lambda_{n}\right\}\left(\lambda_{n} \uparrow+\infty\right)$ as follows:

Let $M_{n}=\max _{|z| \leqq 1}\left|g_{n}(z)\right|$ (note $M_{n}$ is finite since $g_{n}(z)$ is a polynomial). Choose a sequence of positive numbers $\left\{\varrho_{n}\right\}\left(\varrho_{n} \uparrow 1\right)$ such that $p\left(\varrho_{n}\right)>2^{n} M_{n}$ (this is possible since $p(r) \uparrow+\infty)$. Now pick a $\lambda_{n}$ such that $\left|g_{n}\left(z^{\prime \cdot n}\right)\right|<$ $p(|z|) / 2^{n}$ for $0 \leq|z|<1$. 
To see that it is possible to find such a sequence $\lambda_{n}$ we note that all that is needed is to pick a $\lambda_{n}$ large enough that the disk $\left\{z:|z| \leq \varrho_{n}\right\}$ is mapped into the disk $\left\{w:|w| \leq \frac{1}{2}\right\}$ by $w=z^{i}{ }^{i n}$. Now, since $p(r)$ is increasing, we have the following inequalities:

i) for $|z| \leq \varrho_{n}$ :

$$
\begin{aligned}
\left|g_{n}\left(z^{\prime} n\right)\right| & <\varepsilon_{n} \\
& <p(0) / 2^{n} \\
& \leq p(|z|) / 2^{n} .
\end{aligned}
$$

ii) for $\varrho_{n} \leq|z|<1$ :

$$
\begin{aligned}
\left|g_{n}\left(z^{\prime} n\right)\right| & <M_{n} \\
& <p\left(\varrho_{n}\right) / 2^{n} \\
& <p(|z|) / 2^{n} .
\end{aligned}
$$

Now let $g(z)=\sum_{n=1}^{k} g_{n}\left(z^{\prime} n\right)$. This series converges by the Weierstrass $M$-test, to a function holomorphic in $D$ since $\sum_{n=1}^{\infty}\left|g_{n}\left(z^{i} n\right)\right| \leq \sum_{n=1}^{\infty} p(|z|) / 2^{n}=$ $p(|z|)$. It also follows that $|g(z)| \leq p(|z|)$ and hence $M(r, g) \leq p(r)$.

It remains now for us to show that $\operatorname{Re} g(z)$ has the radial limit $-\infty$ on a set of measure $2 \pi$.

Consider $\tilde{R}\left(\delta_{n}\right)$ as a set in the $w$-plane. Let $\tilde{S}\left(\delta_{n}\right)$ be the set in the $z$-plane which is the pre-image of $\tilde{R}\left(\delta_{n}\right)$ under the map $w=z^{\prime} n$. It is clear that meas $\left(\tilde{S}\left(\delta_{n}\right) \cap C\right)=$ meas $\left(\tilde{R}\left(\delta_{n}\right) \cap C\right)$ and hence $2 \pi-\delta_{n}<$ meas $\left(\tilde{S}\left(\delta_{n}\right) \cap C\right)<2 \pi$.

Let $T=\bigcup_{k=1}^{\infty} \bigcap_{n=k}^{\infty} \tilde{S}\left(\partial_{n}\right) \quad$ (note that $T \subset C$ ).

Let $T$ be the set of all unit radii which terminate at a point of $T$. Now consider a fixed radius $\gamma \in \Gamma$.

Let $k_{0}(\geq 2)$ be the first integer $k$ such that $\gamma \cap\left(\cap \tilde{S}\left(\partial_{n}\right)\right) \neq 0$.

Define $M_{0}$ as $M_{0}=\sum_{j=1}^{k_{0}-1} M_{j}\left(k_{0}\right.$ chosen greater than one in order that $M_{0}$ make sense).

Now choose $k_{1}$ such that $\left|\sum_{j=k_{0}}^{k_{1}} \beta_{i}\right|>M_{0}$ (this is possible since $\left.\beta_{i} \downarrow-\infty\right)$.

For any $L>0$ pick $k_{2}$ such that $\left|\sum_{j=k_{1}+1}^{k_{2}} \beta_{k}\right|>L$.

Determine $Q^{*}$ such that:

$$
\gamma^{*}=\gamma \cap\left\{z: 1-Q^{*} \leq|z| \leq 1\right\}
$$

is not mapped into $\left\{w:|w| \leq \frac{7}{8}\right\}$ by the mappings $w=z^{i j}$ for $j=1$, $2, \ldots, k_{2}$. 
For any $z \in \gamma^{*}$ (recalling that the $\beta_{j}$ 's are negative) we have:

$$
\begin{aligned}
\operatorname{Re} g(z)= & \sum_{j=1}^{\infty} \operatorname{Re} g_{j}\left(z^{j j}\right) \\
= & \sum_{j=1}^{k_{0}-1} \operatorname{Re} g_{j}\left(z^{j j}\right)+\sum_{j=k_{0}}^{k_{1}} \operatorname{Re} g_{j}\left(z^{i j}\right) \\
& +\sum_{j=k_{1}+1}^{k_{2}} \operatorname{Re} g_{j}\left(z^{j \cdot j}\right)+\sum_{j=k_{2}-1}^{\infty} \operatorname{Re} g_{j}\left(z^{i j}\right) \\
< & M_{0}+\sum_{j=k_{0}}^{k_{1}}\left(\beta_{j}+\varepsilon_{j}\right)+\sum_{j=k_{1}+1}^{k_{2}}\left(\beta_{j}+\varepsilon_{j}\right)+\sum_{j=k_{2}+1}^{\infty} \operatorname{Re} g_{j}\left(z^{i j}\right) \\
< & M_{0}+\sum_{j=k_{0}}^{k_{1}}\left(\beta_{j}+\varepsilon_{j}\right)+\sum_{j=k_{1}+1}^{k_{2}}\left(\beta_{j}+\varepsilon_{j}\right)+\sum_{j=k_{1}+1}^{\infty} \varepsilon_{j} \quad(\text { since } z \in \gamma) \\
< & M_{0}-M_{0}-L+\sum_{=k_{0}}^{\infty} \varepsilon_{j} \\
\leq & -L+\varepsilon .
\end{aligned}
$$

This implies that if $z_{n} \in \gamma$, then $\lim \operatorname{Re} g\left(z_{n}\right)=-\infty$.

$$
\left|z_{n}\right| \rightarrow 1
$$

Now it remains only for us to prove that meas $T=2 \pi$.

Consider any $\eta>0$. Since $\sum_{n=1}^{\infty} \delta_{n}<1 / 10$, we can find an $\lambda$ such that $\sum_{n=N}^{\infty} \delta_{n}<\eta$. Thus we have:

$$
\begin{aligned}
& \operatorname{meas} T \geq \operatorname{meas}\left(\bigcap_{n=N}^{\infty} \tilde{S}\left(\delta_{n}\right)\right) \\
& =\operatorname{meas}\left[C-\bigcup_{n=N}^{\infty}\left(C-\bar{S}\left(\delta_{n}\right)\right)\right] \\
& =\operatorname{meas} C-\operatorname{meas}\left[\bigcup_{n=N}^{\infty}\left(C-\tilde{S}\left(\partial_{n}\right)\right)\right] \\
& \geq \operatorname{meas} C-\sum_{n=1}^{\infty} \operatorname{meas}\left(C-\tilde{S}\left(\partial_{n}\right)\right) \\
& \geq 2 \pi-\sum_{n=1}^{\infty} o_{n} \\
& >2 \pi-\mu \text {. }
\end{aligned}
$$

Hence meas $T=2 \pi$ which completes the proof of 'Theorem 1 .

Clearly we can obtain an analog of Theorem 1 for harmonic functions just by considering the harmonic function Re $g(z)$.

Theorem 2. Let $\mu(r)$ be a positive function such that $\mu(r) \uparrow+\infty$ as $r \uparrow 1$. Then there exists a nonconstant function $f(z)$ holomorphic in $D$ with the properties that: 
i) $f(z)$ has radial limit zero at each point of $T(\subset C)$ and meas $T=2 \pi$,

ii) $M(r, f)<\mu(r)$.

Proof. First we suppose $\mu(0)>1$. In this case $p(r)=\log \mu(r)$ satisfies the hypothesis of Theorem 1 . Therefore by Theorem 1 there exists a function $g(z)$, holomorphic in $D$, such that the $\operatorname{Re} g(z)$ has radial limit $-\infty$ at almost all points of $C$ and $M(r, g)<p(r)$. Hence $f(z)=e^{g(z)}$ has radial limit zero at almost all points of $C$ and $M(r, f)<\mu(r)$.

If $\mu(0) \leq 1$, then there exists a positive number $k$ such that $k \cdot \mu(0)$ $>1$ and hence by the above argument we can find a function $h(z)$ such that $M(r, h)<k \cdot \mu(r)$. Now clearly $f(z)=h(z) / k$ has the desired properties.

\section{PROOF OF THEOREM 3}

Theorem 3. Let $f(z)$ be a normal meromorphic function in $D$. If $f(z)$ has radial limit zero on a set $T(\subset C)$ of positive measure, then $f^{\prime}(z) \equiv 0$

Proof. Since for normal functions the existence of a radial limit implies the existence of an angular limit ([3], Theorem 2) $f(z)$ has angular limit zero at each point of $M$. Hence by the angular uniqueness theorem of Lusin and Priwalow ([9], p. 212) we have $f(z) \equiv 0$.

\section{REMARKS}

Either by the Lusin-Priwalow radial uniqueness theorem ([9], p. 232) or by direct computation it can be seen that the set of points where the function $f(z)$, in Theorem 2, has radial limit zero is a set of first category on $C$. Since the $R\left(\delta_{n}\right)$ 's are such arbitrary sets (as long as they are metrically large, closed and nowhere dense) we could have proved Theorem 2 not just for a set $T$ of measure $2 \pi$ but $T$ could also have been chosen so as to contain any set $S$ (given in advance) of first category. Hence in this sense Theorem 2 is best possible.

The authors' original proof of Theorem 2 was a very long, involved and highly computational potential-theoretic one. Afterwards the authors noticed that the use of the highly ingenious device of MacLane ([6], p. 22) of precomposing by the map $w=z^{n}$ made it possible to sweep away almost all of their original difficulties.

We might also remark that while there seems to be no easy way of getting Theorem 2 without this device one can still get, without this device, a very 
short and very intuitive proof of MacLane's original theorem [6] on the impossibility of extending the Fatou radial limit theorem to functions of slow growth.

Proof of MacLane's theorem. First one considers any entire function. $f(z)$ which has asymptotic value one on the spiral $r=\Theta+\frac{1}{2}(0 \leq \Theta<\infty)$ and asymptotic value zero on the spiral $r=\Theta+1(0 \leq \Theta<\infty)$ (by Bagemihl and Seidel's Tress theorem ([2], Theorem 1) such functions exist). Now consider a gap series $g(z)=\sum_{n=1}^{\infty} a_{i_{n}} z^{\lambda_{n}}$ with the properties:

i) $M(r, g(z))$ grows so slowly that $M(r, f(g(z)))<\mu(r)$,

ii) The gaps are so large that on a disjoint sequence of concentric annuli $A_{n}$ (approaching $C$ ) $g(z)$ is approximately equal to $a_{i_{n}} z^{\lambda_{n}}$ and $g\left(A_{n}\right)$ contains annuli $B_{n}$ of larger and larger widths (=outer radius - inner radius) as $n$ goes to infinity.

(That such gap series exist is easy to verify.)

Now $h(z)=f(g(z))$ will be of slow growth. Also $h(z)$ has no radial limits since the image of each radius under $f$ cuts each spiral infinitely many times.

Syracuse University

Syracuse, New York

U.S.A. 


\section{References}

1] Bageminl, F., Erdös, P. and Seidel, W. Sur quelques propriétés frontières des fonctions holomorphes définies par certains produits dans le cercle-unité. Ann. Sci. Ecole Norm. Sup. (3) 70, 135- 147 (1953).

[2] - - - and Seidel, W. Some boundary properties of analytic functions. Math. Z. $61,186-199$ (1954).

[3] Lehto, O. and Virtanen, K. I. Boundary behaviour and normal meromorphic functions. Acta Math. 97, 47-65 (1957).

[4] Lohwater, A. J. and Piranian, G. The boundary behavior of functions analytic in a disk. Ann. Acad. Sci. Fenn. Ser. AI, No. 239, 1-17 (1957).

[5] Maclane, G. R. Meromorphic functions with small characteristic and no asymptotic values. Mich. Math. J. 8, 177-185 (1961).

[6] - - Holomorphic functions of arbitrarily slow growth, without radial limits, Mich. Math. J. 9, 21-24 (1962).

[7] Mergelyan, S. N. On the representation of functions by series of polynomials on closed sets (in Russian) Doklady Akad. Nauk SSSR, N.S. vol. 78, 405-408 (1951); Amer. Math. Soc. Transl. No. 85, Providence (1953).

[8] Nevanlinna, R. Eindeutige analytische Funktionen, Zweite Auflage, Springer, Berlin-Göttingen-Heidelberg (1953).

[9] Priwalow, I. I. Randeigenschaften analytischer Funktionen, VEB Deutscher Verlag der Wissenschaften, Berlin (1956). 